\title{
Overexpression and proliferation dependence of acyl-CoA thioesterase 11 and 13 in lung adenocarcinoma
}

\author{
JEN-YU HUNG ${ }^{1,2^{*}}$, SHYH-REN CHIANG ${ }^{3 *}$, KUAN-TING LIU ${ }^{1,4,5}$, MING-JU TSAI ${ }^{2}$, \\ MING-SHYAN HUANG ${ }^{1,2}$, JIUNN-MIN SHIEH ${ }^{3}$, MENG-CHI YEN $^{4}$ and YA-LING HSU ${ }^{6}$ \\ ${ }^{1}$ School of Medicine, College of Medicine; ${ }^{2}$ Division of Pulmonary and Critical Care Medicine, Department \\ of Internal Medicine, Kaohsiung Medical University Hospital, Kaohsiung $807 ;^{3}$ Department of Internal \\ Medicine, Chi Mei Medical Center, Tainan 710; ${ }^{4}$ Department of Emergency Medicine, Kaohsiung \\ Medical University Hospital; ${ }^{5}$ Institute of Clinical Medicine, College of Medicine; ${ }^{6}$ Graduate Institute of \\ Medicine, College of Medicine, Kaohsiung Medical University, Kaohsiung 807, Taiwan, R.O.C.
}

Received December 15, 2015; Accepted May 8, 2017

DOI: $10.3892 / 01.2017 .6594$

\begin{abstract}
The metabolites of fatty acyl-Coenzyme A (CoA) and metabolic enzymes contribute to lipid biosynthesis, signal transduction, and gene transcription. Previous studies have indicated that elevated concentrations of specific free fatty acids in the plasma and overexpression of specific fatty acyl-CoA metabolic enzymes are observed in patients with lung adenocarcinoma. However, there are $>30$ enzymes in this metabolic network and have been fully investigated. In the present study, the expression levels of enzymes in the acyl-CoA synthetase (ACS) and acyl-CoA thioesterase (ACOT) families were analyzed from six microarray expression datasets that were collected from Gene Expression Omnibus. Compared with adjacent non-tumor lung tissue, lung adenocarcinoma tissue exhibited significantly higher ACOT11 and ACOT13 expression. Kaplan-Meier plotter database analysis demonstrated that high levels of ACOT11 and ACOT13 were associated with a worse overall survival rate. The proliferation of the lung adenocarcinoma cell lines CL1-0 and CL1-5 was inhibited when ACOT11 and ACOT13 were downregulated by short hairpin RNA. Although ACOT11 and ACOT13 knockdown did not significantly affect the total amount of intracellular and medium-free fatty acids, ACOT11 and ACOT13 knockdown-mediated growth inhibition was
\end{abstract}

Correspondence to: Professor Ya-Ling Hsu, Graduate Institute of Medicine, College of Medicine, Kaohsiung Medical University, 100 Shih-Chuan 1st Road, Kaohsiung 807, Taiwan, R.O.C.

E-mail: hsuyl326@gmail.com

Dr Meng-Chi Yen, Department of Emergency Medicine, Kaohsiung Medical University Hospital, Kaohsiung Medical University, 100 Tzyou 1st Road, Kaohsiung 807, Taiwan, R.O.C.

E-mail:yohoco@gmail.com

${ }^{*}$ Contributed equally

Key words: acyl-CoA thioesterase 11 (ACOT11), acyl-CoA thioesterase 13 (ACOT13), lung adenocarcinoma, lipid metabolism rescued by the addition of fatty acids. In conclusion, ACOT11 and ACOT13 were upregulated in clinical specimens of lung adenocarcinoma, which may contribute to increased cell proliferation through the increased availability of fatty acids. The metabolites of the two enzymes may be critical for development of lung adenocarcinoma.

\section{Introduction}

Metabolites are currently considered targets for cancer treatments, particularly amino acids and glucose (1). Fatty acyl-Coenzyme A (CoA) esters are essential components in lipid metabolism and are regulators of multiple cellular functions (2). Enzymes of the acyl-CoA synthetase (ACS) family, including the ACS long-chain, ACS medium-chain, ACS short-chain and ACS bubblegum families, ligate different lengths of fatty acid with CoA. Fatty acyl-CoA esters are hydrolyzed into free fatty acids and CoA by enzymes of the acyl-CoA thioesterase (ACOT) family $(3,4)$. There are $>15$ ACOT enzymes and 20 ACS enzymes in humans, and these enzymes exhibit different tissue distribution, subcellular location and substrate specificity $(3,4)$.

Lung cancer is a leading cause of cancer-associated mortality and is the second most commonly diagnosed cancer (5). The majority ( $85-90 \%)$ of diagnosed cases of lung cancer are diagnosed as non-small cell lung cancer (NSCLC) and adenocarcinoma is the most common subtype of NSCLC (6). Compared with healthy individuals, patients with lung adenocarcinoma possess a significantly higher level of fatty acids (including arachidonic, palmitic, linoleic and oleic acid) in their plasma $(7,8)$. In addition, overexpression of ACOT8 is associated with metastasis in lung adenocarcinoma (9). However, the cellular functions and the regulatory mechanisms of the majority of ACOT and ACS enzymes in lung adenocarcinoma remain unclear. In the present study, in order to systematically analyze the expression pattern of these enzymes, expression levels of ACOT and ACS enzymes were analyzed in clinical specimens of lung adenocarcinoma from six microarray datasets that were collected from an online database. In addition, the effect of these enzymes and their 
metabolic products on cell proliferation was measured in lung adenocarcinoma cell lines.

\section{Materials and methods}

Collection of microarray datasets of human lung adenocarcinoma specimens. Expression profiling microarray data of human lung adenocarcinoma clinical specimens, which was published between 2005 and 2015, was collected from the National Center for Biotechnology Information Gene Expression Omnibus (GEO, http://www.ncbi.nlm.nih.gov/geo/) (10). Microarray data from cell lines or small sample sizes $(<10$ samples) were excluded. Six microarray datasets including GSE2514 (11), GSE7670 (12), GSE10072 (13), GSE31210 (14), GSE32863 (15), and GSE43458 (16) were collected and the relative expression levels of fatty acyl-CoA metabolic enzymes between adjacent non-tumor lung tissue and lung adenocarcinoma were analyzed in these microarray datasets. The expression value was analyzed using the GEO2R interface (http://www.ncbi.nlm.nih.gov/geo/geo2r/).

Kaplan Meier (KM)-Plotter. The survival analysis in lung adenocarcinoma patients with different expression levels of ACOT11 and ACOT13 was performed using the KM-Plotter database (17). The prognostic value of each gene was analyzed by splitting patient samples into two groups according to median expression. After the subtype of lung cancer was restricted ('Histology: adenocarcinoma') and survival rate was analyzed through the '2015 version' database and 'excluded biased array', 720 patients were analyzed.

Chemicals. Dimethyl sulfoxide (DMSO), puromycin, myristic acid, palmitic acid and stearic acid were purchased from Sigma-Aldrich (Merck KGaA, Darmstadt, Germany). Myristic acid, and palmitic acid and stearic acid were dissolved in DMSO.

Cell culture. Human lung adenocarcinoma cell lines CL1-0 and CL1-5 were provided by Dr Pan-Chyr Yang (Department of Internal Medicine, National Taiwan University Hospital, Taipei, Taiwan) and were cultured in RPMI-1640 supplemented with $10 \%$ fetal bovine serum and $1 \%$ penicillin-streptomycin (all Thermo Fisher Scientific, Inc., Waltham, MA, USA) in a humidified incubator at $37^{\circ} \mathrm{C}$ with $5 \% \mathrm{CO}_{2}(18,19)$.

Short hairpin (sh)RNA and transfection. shRNA targeting ACOT11 (shACOT11; TRCN0000048912; targeting sequence: 5'-GTCTCCTCCTTGAAGATGCT-3'), ACOT13 (shACOT13-1; TRCN0000048954; targeting sequence:5'-CGA TATGAACATAACGTACAT-3'; shACOT13-2; targeting sequence: TRCN0000048956; targeting sequence: 5'-GAA GAGCATACCAATGCAATA-3') and a negative control construct (luciferase shRNA, shLuc) were obtained from the National Core Facility for Manipulation of Gene Function by RNAi, miRNA, miRNA sponges, and CRISPR/Genomic Research Center, Academia Sinica (Taipei, Taiwan). CL1-0 and CL1-5 cells were transfected with each shRNA plasmid using Lipofectamine ${ }^{\circledR} 2000$ reagent (Invitrogen; Thermo Fisher Scientific, Inc.) according to the manufacturer's protocol. Transfected cells were selected and maintained in medium containing $2 \mu \mathrm{g} / \mathrm{ml}$ puromycin.
Reverse transcription-quantitative polymerase chain reaction (RT-qPCR). Total RNA was extracted from cells using TRIzol reagent (Invitrogen; Thermo Fisher Scientific, Inc.) according to manufacturer's protocol. Complementary DNA (cDNA) was reverse transcribed from mRNA using the PrimeScript RT reagent kit (Clontech Laboratories, Inc., Mountainview, CA, USA) according to the manufacturer's protocol. PCR was performed using the following primers: ACOT13 forward, 5'-TCTGCTATGCACGGAAAGGG-3' and reverse, 5'-TTTCCTGTGGCCTTGTTGGT-3'; ACOT11 forward, 5'- GCGATCTGGAGAGCAGAGAC-3' and reverse, 5'-GTGGCCACATTCTCCATCCA-3'; GAPDH forward, 5'-GAGTCAACGGATTTGGTCGT-3' and reverse, 5'-TTG ATTTTGGAGGGATCTCG-3'. PCR was performed on a StepOne Plus Real-Time PCT System (Applied Biosystems; Thermo Fisher Scientific, Inc.) using the Fast SYBR Green Master Mix (Applied Biosystems; Thermo Fisher Scientific, Inc.). The following settings were used: 1 cycle of $95^{\circ} \mathrm{C}$ for $20 \mathrm{sec}$, and 40 cycles of $95^{\circ} \mathrm{C}$ for $3 \mathrm{sec}$ and $60^{\circ} \mathrm{C}$ for $30 \mathrm{sec}$. The mRNA expression levels were normalized to the expression level of GAPDH using the $2^{-\Delta \Delta C q}$ method (20).

Western blot analysis. Cells were lysed in radioimmunoprecipitation assay buffer (EMD Millipore, Billerica, MA, USA) and the total cell lysate was collected by centrifugation at $4^{\circ} \mathrm{C}$, $12,000 \mathrm{x} \mathrm{g}$ for $15 \mathrm{~min}$. Quantification of protein concentration was performed using a BCA protein assay kit (EMD Millipore). A total of $30 \mu \mathrm{g} /$ lane protein was loaded, subjected to $10 \%$ SDS-PAGE and transferred to polyvinylidene difluoride membranes (EMD Millipore). Membranes were blocked with $5 \%$ dried skimmed milk in Tris-buffered saline with Tween-20 buffer and then incubated with the primary antibodies overnight at $4^{\circ} \mathrm{C}$. Protein expression was detected using the following primary antibodies: Anti-ACOT11 (dilution, 1:3,000; cat. no. ab153835), anti-ACOT13 (dilution, 1:1,000; cat. no. ab166684; both Abcam, Cambridge, UK) and anti-GAPDH (dilution, 1:6,000; cat. no. MAB374; EMD Millipore). Each membrane was then incubated with secondary andibodies, including peroxidase conjugated goat anti-rabbit IgG (dilution, 1:5,000; cat. no. AP132P) and peroxidase conjugated goat anti-mouse IgG, (dilution, 1:5,000; cat. no. AP124P; both EMD Millipore) at room temperature for $1 \mathrm{~h}$. Immunoreactive signals were detected with Immobilon horseradish peroxidase substrate enhanced chemiluminescence reagents (EMD Millipore) and analyzed with the Alpha Innotech FluorChem FC2 imaging system (ProteinSimple; Bio-Techne, Minneapolis, MN, USA).

Cell proliferation assay. For cell proliferation measurements, WST-1 (Clontech Laboratories, Inc.) was used. A total of $5 \times 10^{3}$ CL1-0 or $2.5 \times 10^{3}$ CL1-5 cells were seeded in 96-well plates. The proliferation rate was determined at a wavelength of $450 \mathrm{~nm}$ on a microplate spectrophotometer (PowerWave X340, BioTek Instruments, Inc., Winooski, VT, USA).

Free fatty acid quantification. A total of $1 \times 10^{6}$ CL1-0 cells were seeded into $10 \mathrm{~cm}$ dishes with $8 \mathrm{ml}$ of culture medium. After $48 \mathrm{~h}$, culture medium and cells were collected for free fatty acid quantification. Free fatty acids released into the culture medium and intracellular free fatty acid were 
Table I. mRNA expression of ACOT enzymes in lung adenocarcinoma tissue compared with normal tissue, from the GEO database.

Variable $\quad \begin{gathered}\text { Expression ratio } \\ \text { (normal/tumor) }\end{gathered}$ P-value Probe

Adjacent non-tumor ( $n=19)$ vs. Lung adenocarcinoma $(n=20)$;

GEO accession number: GSE2514; GPL8300 platform (11)

ACOT1/ACOT2
ACOT7
ACOT8
ACOT11
ACOT13

Adjacent non-tumor $(n=26)$ vs. Lung adenocarcinoma $(n=26)$;

GEO accession number: GSE7670; GPL96 platform (12)

ACOT1/ACOT2
ACOT7
ACOT8
ACOT9
ACOT11
ACOT13
Non-tumor tissue (49) vs. Lung adenocarcinoma (58);
GEO accession number: GSE10072; GPL96 platform (13)
ACOT1/ACOT2
ACOT7
ACOT8
ACOT9
ACOT11
ACOT13

Normal lung tissue $(n=20)$ vs. Lung adenocarcinoma $(n=226)$;

GEO accession number: GSE31210; GPL570 platform (14)

ACOT2
ACOT4
ACOT6
ACOT7
ACOT8
ACOT9
ACOT11
ACOT12
ACOT13

1.141

0.823

0.863

0.880

0.715

0.861

0.843

1.120

0.564

0.678

1.037

0.991

1.002

1.010

0.976

0.934

0.699

1.122

0.693

0.681

0.981

0.256

1.007

0.549

Adjacent non-tumor ( $\mathrm{n}=58)$ vs. Lung adenocarcinoma $(\mathrm{n}=58)$;

GEO accession number: GSE32863; GPL6884 platform (15)

ACOT1

ACOT2

ACOT4

ACOT6

ACOT7

ACOT8

ACOT9

ACOT11

ACOT12

ACOT13

Normal lung tissure (30) vs. Lung adenocarcinoma (80);

GEO accession number: GSE43458; GPL6244 platform (16)
1.012

1.007

0.998

0.999

0.998

1.004

0.991

0.879

1.005

0.963

$$
\begin{array}{r}
0.2120 \\
0.0279 \\
0.1003 \\
0.3971 \\
<0.0001
\end{array}
$$

0.5266

0.1354

0.1336

0.8513

0.0002

0.0004

0.0002

0.4437

0.3531

0.1675

0.0001

$<0.0001$

0.7030

0.0215

0.6088

0.7152

0.0338

0.7506

0.0017

0.9543

$<0.0001$

0.0049

0.0065

0.8718

0.7702

0.5223

0.5715

0.2383

$<0.0001$

0.0271

0.0010 36625_at

37945_at

36841_at

32405_at

41058_g_at

202982_s_at 208002_s_at 204212_at

221641_s_at

214763_at

204565_at

202982_s_at 208002_s_at 204212_at 221641_s_at 214763_at 204565_at

$$
\begin{aligned}
& \text { 202982_s_at } \\
& 229534 \text { at } \\
& 241949 \text { at } \\
& \text { 208002_s_at } \\
& 236514 \text { at } \\
& \text { 221641_s_at } \\
& 214763 \text { at } \\
& 238160 \text { at } \\
& 204565 \text { at }
\end{aligned}
$$

ILMN_2121389

ILMN_2188959

ILMN_1764321

ILMN_2156699

ILMN_1719178

ILMN_1679600

ILMN_2367070

ILMN_1739594

ILMN_1785474

ILMN_2098743 
Table I. Continued.

\begin{tabular}{lcrc}
\hline Variable & $\begin{array}{c}\text { Expression ratio } \\
\text { (normal/tumor) }\end{array}$ & P-value & 0.0794 \\
\hline ACOT4 & 0.982 & 0.0810 & 7975607 \\
ACOT6 & 0.987 & 0.0095 & 7975613 \\
ACOT7 & 0.960 & 0.1137 & 8066598 \\
ACOT8 & 1.010 & $<0.0001$ & 8171802 \\
ACOT9 & 1.052 & $<0.0001$ & 7901613 \\
ACOT11 & 0.952 & 0.4263 & 8112920 \\
ACOT12 & 0.995 & 0.0039 & 8117219 \\
\hline
\end{tabular}

GEO, Gene Expression Omnibus; ACOT, acyl-CoA thioesterase.

quantified using a Free Fatty Acid Quantification Colorimetric/Fluorometric kit (BioVision, Inc., Milpitas, CA, USA) according to the manufacturer's protocol. The results were determined on a fluorescent microplate reader at excitation/emission=485/590 nm (FLX800 Microplate Fluorescence Reader; BioTek Instruments, Inc.).

Statistical analysis. All statistical analyses were performed using GraphPad Prism software (version 5.03; GraphPad Software, Inc., La Jolla, CA, USA). All error bars in the figures represent standard error of the mean. The differences between two independent groups were analyzed using a Student's t-test. A log-rank test was performed to assess differences in survival rate. $\mathrm{P}<0.05$ was considered to indicate a statistically significant difference.

\section{Results}

High ACOT11 and ACOT13 expression is associated with lung adenocarcinoma and poor overall survival rate. To investigate whether the expression of ACSs and ACOTs was associated with lung adenocarcinoma, microarray datasets with lung adenocarcinoma specimens were collected from the GEO database. Following the exclusion of microarray data regarding cell line studies and small sample sizes ( $<10$ samples), six microarrays that were performed by five different array platforms were selected for the current study (Fig. 1). A number of enzymes were significantly differentially expressed in lung adenocarcinoma tissue compared with non-tumor lung tissue (Tables I and II). ACOT11 and ACOT13 overexpression was observed in lung adenocarcinoma tissue across all selected microarray datasets (Table I). A KM-Plotter database was used to further investigate the association between the expression of ACOT11 and ACOT13 and clinical outcome. Poor overall survival rate of patients with lung adenocarcinoma was associated with high expression of ACOT11 and ACOT13 (P<0.001; Fig. 2). These results suggest that ACOT11 and ACOT13 serve roles in the development of lung adenocarcinoma.

ACOT11 and ACOT13 knockdown decreases cell proliferation but does not affect the level of free fatty acids. The
GEO database: find dataset containing

Human Lung adenocarcinoma and normal Lung tissue (More than 10 samples, without drug treatment)

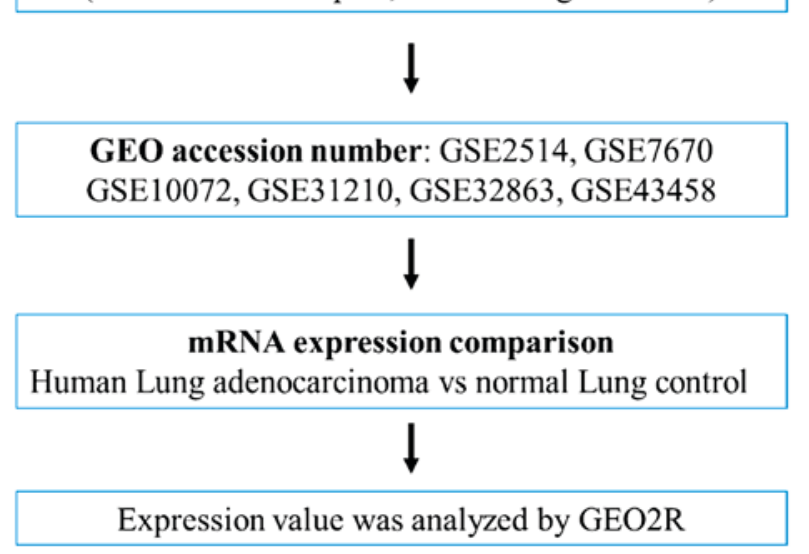

Figure 1. Scheme of microarray dataset collection. Six datasets were collected from the GEO database. GEO, Gene Expression Omnibus.

CL1-0 and CL1-5 cell lines were established from a patient with lung adenocarcinoma and exhibit different invasive and metastatic properties (19). Similar protein levels of ACOT11 and ACOT13, and similar levels of intracellular and medium free fatty acid, were observed in the two cell lines (Fig. 3A and B). In order to determine the role of ACOT11 and ACOT13, shRNAs targeting ACOT11 (shACOT11) and ACOT13 (shACOT13-1 and shACOT13-2) were transfected into CL1-0 and CL1-5 cells. Cells treated with shACOT11 exhibited significantly decreased expression of ACOT11 (P<0.05; Fig. 4A). Similarly, cells treated with shACOT13-1/-2 exhibited significantly decreased expression of ACOT13 $(\mathrm{P}<0.01$ and $\mathrm{P}<0.001$, respectively; Fig. 4A). These changes were also exhibited at the protein level (Fig. 4B). Although decreasing expression of ACOT11 and ACOT13 did not result in a significant change in total free fatty acids (Fig. 4C), significantly decreased proliferation rates were observed in CL1-0 and CL1-5 cells treated with shACOT11 compared with the control $(\mathrm{P}<0.001$ and $\mathrm{P}<0.01$, respectively; Fig. 4D). In addition, CL1-0 cells treated with shACOT13-2, and 

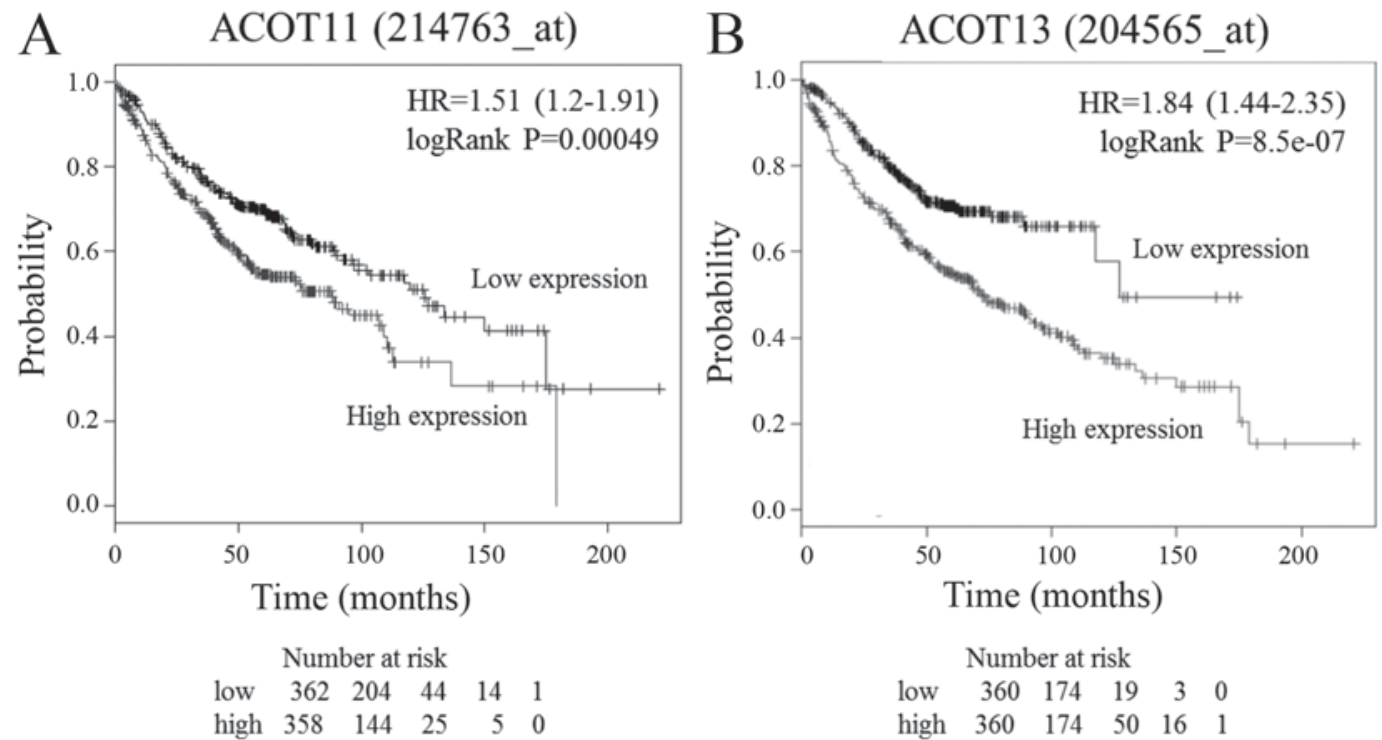

Figure 2. Kaplan-Meier analysis of ACOT11 and ACOT13 levels and survival rate of patients with lung adenocarcinoma. (A) Overall survival in low ACOT11 expression group and high ACOT11 expression groups. (B) Overall survival in low ACOT13 expression group and high ACOT13 expression group. KM-plotter database was used for analyses (2015 version). The probe for ACOT11 (Affymetrix ID: 214763_at) and for ACOT13 (Affymetrix ID: 204565_at) were used. Log-rank test was used for statistical significance. ACOT, acyl-CoA thioesterase; HR, hazard ratio.
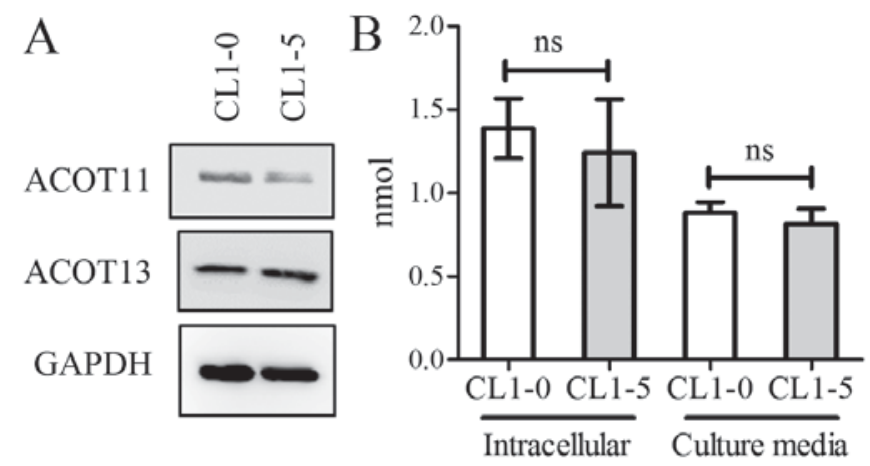

Figure 3. ACOT expression and free fatty acid level in lung adenocarcinoma cell lines. (A) Protein expression of ACOT11 and ACOT13 in CL1-0 and CL1-5 cells. (B) Levels of intracellular and medium free fatty acids in CL1-0 and CL1-5 cells. Data is presented as the mean \pm standard error of the mean. ACOT, acyl-CoA thioesterase; ns, not significant.

CL1-5 cells treated with ahACOT13-1 exhibited significantly decreased proliferation rates compared with the control $(\mathrm{P}<0.001$ and $\mathrm{P}<0.05$, respectively; Fig. 4D). These results suggest that ACOT11 and ACOT13 are critical for proliferation in lung adenocarcinoma cell lines.

Free fatty acid supplements rescue the decreased proliferation rate induced by ACOT11 and ACOT13 knockdown. ACOT11 and ACOT13 are members of the Type II ACOT family (21). The substrates of ACOT11 and ACOT13 include medium (6-12 carbon) to long (13-21 carbon) chain acyl CoA (22). It was hypothesized that the observed decreased proliferation rates following ACOT11/13 knockdown were associated with insufficient availability of free fatty acids. Therefore ACOT11/13 knockdown CL1-0 cells were treated with a fatty acid mixture, including myristic (C14:0), palmitic (C16:0) and stearic acid (C18:0). The cell proliferation rate was significantly decreased in the control groups (medium and vehicle) following treatment with shACOT11/13-2 compared with the control luciferase shRNA group ( $\mathrm{P}<0.05$; Fig. 5); however, treatment with the fatty acid mixture $(0.1-10 \mu \mathrm{M})$ restored shACOT11 and shACOT13-mediated growth inhibition. However, this effect was abolished following the addition of mixed free fatty acids at the highest dose $(100 \mu \mathrm{M})$, which may indicate a negative feedback mechanism. The results suggest that the proliferation of lung adenocarcinoma cells is dependent on ACOT11 and ACOT13-mediated metabolic products.

\section{Discussion}

Dysregulation of metabolism is a hallmark of cancer development. Acyl-CoAs are involved in the biosynthesis of lipids, signal transduction and gene transcription (23). Previous studies have demonstrated that certain types of fatty acid are increased in the serum or plasma of patients with lung adenocarcinoma $(7,8)$. Notably, the level of fatty acids (palmitic, stearic, oleic, linoleic, arachidonic and palmitoleic acid) decreased in the serum of patients with lung cancer compared with the healthy controls (24). This suggests that different types of lung cancer may possess unique metabolic networks. Although previous studies have demonstrated associations between a single enzyme and a specific tumor type, including the association between ACSL3, ACOT8 and liver cancer $(25,26)$, very long-chain ACS-3 (ACSVL3) and lung cancer (27), and ACOT8 and metastatic lung adenocarcinoma (9), the associations between types of tumor and the enzyme network of acyl-CoA and acyl-CoA esters remain unclear. To systematically determine the potential roles of all ACS and ACOT enzymes in lung adenocarcinoma, the gene expression values from six different microarray datasets were analyzed using five different platforms. Several enzymes exhibited significantly different expression levels between normal and lung adenocarcinoma tissue. High expression of ACOT11 and ACOT13 was observed in tumors compared with normal tissue in each dataset. KM-Plotter 
Table II. mRNA expression of ACS enzymes in lung adenocarcinoma tissue compared with normal tissue, from the GEO database.

\begin{tabular}{lll}
\hline Variable & $\begin{array}{c}\text { Expression ratio } \\
\text { (normal/tumor) }\end{array}$ P-value Probe \\
\hline
\end{tabular}

Adjacent non-tumor ( $n=19)$ vs. Lung adenocarcinoma $(n=20)$;

GEO accession number: GSE2514; GPL8300 platform (11)

ACSBG1
ACSM1
ACSM2A
ACSM3
ACSL1
ACSL3
ACSL4
ACSL6

$\begin{array}{llc}0.838 & 0.4937 & 32537 \_ \text {at } \\ 0.851 & 0.6678 & 34050 \_ \text {at } \\ 1.120 & 0.6902 & 37800 \_r \text { at } \\ 0.707 & 0.0260 & 33280 \_r \text { at } \\ 1.163 & 0.1418 & 40082 \text { at } \\ 0.941 & 0.6506 & 33880 \_ \text {at } \\ 1.172 & 0.1663 & \text { 38099_r_at } \\ 1.083 & 0.7825 & \text { 31834_r_at }\end{array}$

Adjacent non-tumor ( $n=26)$ vs. Lung adenocarcinoma $(n=26)$;

GEO accession number: GSE7670; GPL96 platform (12)

ACSBG1
ACSBG2
ACSF2
ACSM1
ACSM2A/ACSM2B
ACSM3
ACSM5
ACSS3
ACSL1
ACSL3
ACSL4
ACSL5
ACSL6

0.901
0.892
0.861
0.641
0.667
0.587
1.450
1.098
1.154
1.391
1.240
0.822
0.852

0.7335
0.4825
0.3332
0.1782
0.0532
0.0023
0.0357
0.3422
0.2702
$<0.0001$
0.0936
0.1353
0.0875

206466_at
221716_s_at
218844_at
215432_at
214069_at
210377_at
220061_at
219616_at
207275_s_at
201660_at
202422_s_at
218322_s_at
216409_at

Non-tumor tissue (49) vs. Lung adenocarcinoma (58);

GEO accession number: GSE10072; GPL96 platform (13)

ACSBG1
ACSBG2
ACSF2
ACSM1
ACSM2A/ACSM2B
ACSM3
ACSM5
ACSS3
ACSL1
ACSL3
ACSL4
ACSL5
ACSL6

1.025

1.016

0.989

0.998

1.003

1.013

1.054

1.038

1.041

1.052

1.073

1.026

1.000

Normal lung tissue ( $n=20)$ vs. Lung adenocarcinoma $(n=226)$;

GEO accession number: GSE31210; GPL570 platform (14)

ACSBG1
ACSBG2
ACSF1
ACSF2
ACSM1
ACSM2B
ACSM3
ACSM5

2.087

1.030

0.712

0.653

0.716

1.052

0.422

1.006

0.0007
0.0283
0.1715
0.8514
0.6717
0.2957
$<0.0001$
$<0.0001$
0.0003
$<0.0001$
$<0.0001$
0.0613
0.9467

206466_at 221716_s_at 218844_at 215432_at 214069_at 210377_at 220061_at 219616_at 207275_s_at 201660_at 202422_s_at 218322_s_at 216409_at

206465_at 221716_s_at 218434_s_at 218844_at 215432_at 214069_at 205942_s_at 1554514_at 
Table II. Continued.

\begin{tabular}{|c|c|c|c|}
\hline Variable & $\begin{array}{c}\text { Expression ratio } \\
\text { (normal/tumor) }\end{array}$ & P-value & Probe \\
\hline ACSS1 & 0.767 & 0.0995 & 234484_s_at \\
\hline ACSS2 & 0.857 & 0.3373 & 234312_s_at \\
\hline ACSS3 & 1.220 & 0.1480 & 219616_at \\
\hline ACSL1 & 1.133 & 0.2698 & 207275_at \\
\hline ACSL3 & 0.826 & 0.0403 & 201661_s_at \\
\hline ACSL4 & 1.701 & $<0.0001$ & 1557419_a_at \\
\hline ACSL6 & 0.814 & 0.1151 & 211207_s_at \\
\hline \multicolumn{4}{|c|}{ Adjacent non-tumor $(n=58)$ vs. Lung adenocarcinoma $(n=58)$; } \\
\hline \multicolumn{4}{|c|}{ GEO accession number: GSE32863; GPL6884 platform (15) } \\
\hline ACSBG1 & 1.022 & 0.0072 & ILMN_2227011 \\
\hline ACSBG2 & 1.011 & $<0.0001$ & ILMN_1730002 \\
\hline ACSF1 & 0.953 & $<0.0001$ & ILMN_1698554 \\
\hline ACSF2 & 0.955 & 0.0007 & ILMN_1711928 \\
\hline ACSM1 & 1.000 & 0.9545 & ILMN_1661434 \\
\hline ACSM2A & 1.003 & 0.1501 & ILMN_1754517 \\
\hline ACSM2B & 0.999 & 0.6416 & ILMN_1765912 \\
\hline ACSM3 & 0.960 & $<0.0001$ & ILMN_1662738 \\
\hline ACSM4 & 0.999 & 0.6885 & ILMN_1791923 \\
\hline ACSM5 & 1.004 & 0.0358 & ILMN_1801698 \\
\hline ACSL1 & 1.068 & $<0.0001$ & ILMN_1684585 \\
\hline ACSL3 & 0.985 & 0.0964 & ILMN_2360605 \\
\hline ACSL4 & 0.999 & 0.6174 & ILMN_1691714 \\
\hline ACSL5 & 0.978 & 0.1879 & ILMN_2370882 \\
\hline \multicolumn{4}{|c|}{ Normal lung tissue (30) vs. Lung adenocarcinoma (80); } \\
\hline \multicolumn{4}{|c|}{ GEO accession number: GSE43458; GPL6244 platform (16) } \\
\hline ACSBG1 & 0.994 & 0.4965 & 7990683 \\
\hline ACSBG2 & 0.997 & 0.7000 & 8025011 \\
\hline ACSF2 & 1.021 & 0.1409 & 8008321 \\
\hline ACSF3 & 0.980 & 0.0043 & 7997863 \\
\hline ACSM1 & 0.991 & 0.2103 & 7999981 \\
\hline ACSM2A/ACSM2B & 0.971 & 0.0021 & 7993737 \\
\hline ACSM3 & 0.973 & 0.3288 & 7993756 \\
\hline ACSM5 & 1.008 & 0.3576 & 7993726 \\
\hline ACSM6 & 1.026 & 0.0003 & 7929497 \\
\hline ACSS1 & 1.021 & 0.0387 & 8065444 \\
\hline ACSS2 & 1.040 & 0.0009 & 8062041 \\
\hline ACSS3 & 1.181 & $<0.0001$ & 7957386 \\
\hline ACSL1 & 1.047 & 0.0012 & 8103951 \\
\hline ACSL3 & 1.007 & 0.4760 & 8048733 \\
\hline ACSL4 & 1.067 & $<0.0001$ & 8174474 \\
\hline ACSL5 & 0.970 & 0.0587 & 7930498 \\
\hline ACSL6 & 0.976 & 0.0021 & 8113938 \\
\hline
\end{tabular}

GEO, Gene Expression Omnibus; ACS, acyl-CoA synthetase.

analysis demonstrated that high ACOT11/13 expression was correlated with poor overall survival rate. Since the criteria was not restricted to metastatic lung adenocarcinoma, ACOT8 expression levels in tumor tissue were not significantly different compared with normal tissue in the present study. These results indicated that ACOT11 and ACOT13 enzymes are potential oncogenes in lung adenocarcinoma.

ACOT11 is highly expressed in brown adipose tissue compared with other tissues (28). The ACOT11 structure comprises two 'hotdog' domains and a C-terminal 
A
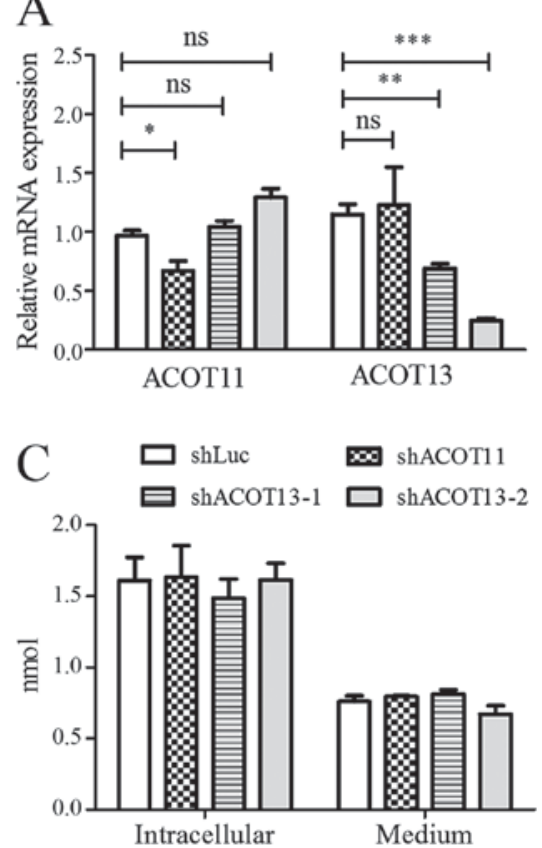

$\mathrm{B}$
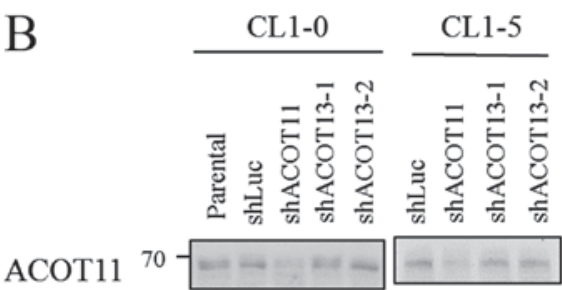

$\operatorname{ACOT} 133^{15}$
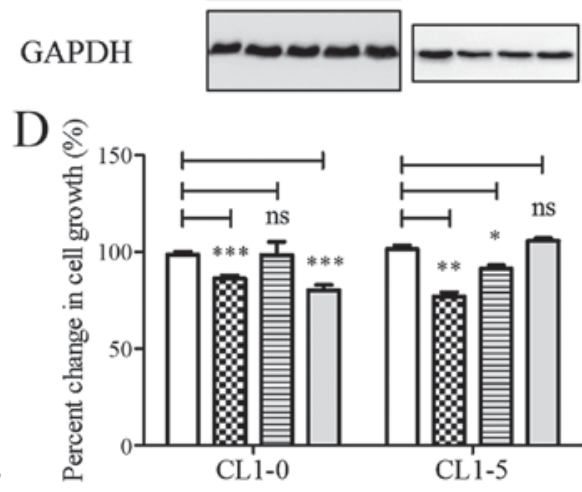

Figure 4. Effect of ACOT11 and ACOT13 knockdown on proliferation rate and biosynthesis of free fatty acids. (A) Relative mRNA expression levels of ACOT11/13, (B) protein expression levels of ACOT11/13 (C) levels of intracellular and medium free fatty acids and (D) proliferation rate of CL1-0 and CL105 cells following treatment with ACOT11 or ACOT13 shRNA. Data is presented as the mean \pm standard error of the mean. ${ }^{*} \mathrm{P}<0.05$, ${ }^{* *} \mathrm{P}<0.01$ and ${ }^{* * * *} \mathrm{P}<0.001$ compared with the control (shLuc) group). ACOT, acyl-CoA thiotransferase; ns, not significant; shRNA, short hairpin RNA; shLuc, luciferase shRNA.

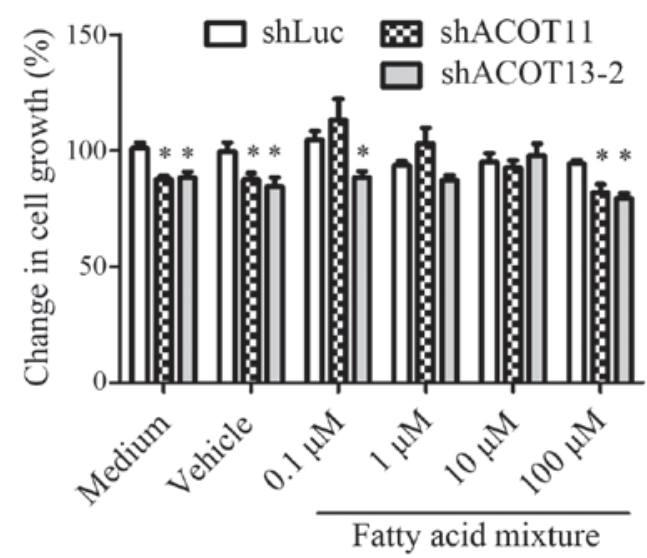

Figure 5. Effect of additional saturated fatty acid supplements with on cell proliferation rate. A total of $5 \times 10^{3}$ CL1-0 cells were seeded in a 96 well plate. Culture medium was replaced with medium containing vehicle $(0.1 \%$ dimethyl sulfoxide) and mixed saturated fatty acid (myristic, palmitic and stearic acid) after $24 \mathrm{~h}$. A WST-1 assay was performed over a $48 \mathrm{~h}$ treatment period. Data is presented as the mean \pm standard error of the mean. ${ }^{*} \mathrm{P}<0.05$ compared with the control (shLuc) group. ACOT, acyl-CoA thioesterase; ns, not significant; shLuc, luciferase shRNA.

lipid-binding steroidogenic acute regulatory transfer-related (START) domain (22). Although a previous study suggested that the START domain may be an important regulatory element for ACOT11 (29), the exact mechanisms underlying the regulation and function of ACOT11 remain unknown in lung adenocarcinoma and other tissues. ACOT11 knockout mice revealed increased energy consumption and resistance to high fat diet-induced obesity compared with control mice (30). In addition, loss of ACOT11 leads to resistance to obesity-induced inflammation and endoplasmic reticulum stress (30). A high

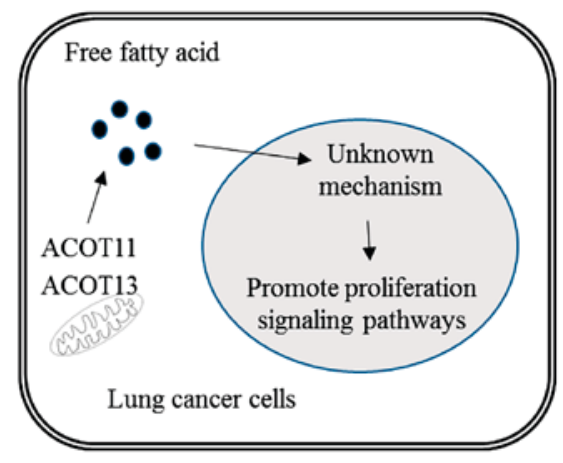

Figure 6. The metabolic products of ACOT11 and ACOT13 are important regulators for lung adenocarcinoma.

fat diet significantly induced ACOT11 expression in mouse liver (3). These observations suggest that ACOT11 expression may serve as a risk factor for obesity. The results from the present study demonstrate that ACOT11 expression is associated with cell proliferation, suggesting that inhibition of ACOT11 may be a strategy to treat lung adenocarcinoma.

AOCT13 comprises a single 'hotdog' domain and is expressed in a number of tissues, including liver, heart, kidney and brown adipose tissue (3). In ACOT13 knockout mice, increasing concentrations of long-chain fatty acyl-CoA and decreasing concentrations of free fatty acids is detected in the liver (31). When mice are fed with a high-fat diet, ACOT13 knockout mice resist increases in glucose production in the liver (31). This observation suggests that ACOT13 serves a role in the regulation of lipid and glucose metabolism in the liver. Notably, ACOT13 interacts with phosphatidylcholine transfer protein (PC-TP), which possesses a START domain (32). 
Addition of recombinant PC-TP increases ACOT13 enzyme activity in vitro (32). The interaction affects the transcriptional activity of peroxisome proliferator-activated receptor alpha and hepatocyte nuclear factor 4 alpha in the liver (33). Since ACOT11 contains a START domain, these observations imply that ACOT13 may also interact with ACOT11. Since overexpression of ACOT11 and ACOT13 was observed in the present study, these interactions may regulate critical biological functions in lung adenocarcinoma.

The level of free fatty acids is significantly altered in brown adipose tissue and liver in ACOT11 and ACOT13 knockout mice, respectively $(30,31)$. However, in the present study, ACOT11 and ACOT13 knockdown did not affect the level of total amount of free fatty acid in CL1-0 cells. There are two possible reasons for this. First, ACOT11 and ACOT13 may not be major lipid-metabolic enzymes in the lung adenocarcinoma cell. Decreasing levels of ACOT11 and ACOT13-hydrolyzed free fatty acids (medium to long-chain) may account for a small part of the total free fatty acid pool. Second, free fatty acids may be adequately supplied from the culture medium. Although ACOT11 and ACOT13 knockdown results in the reduction of certain types of free fatty acid, the effect may be diluted in the free fatty acid pool. Addition of free fatty acid mixture restored the growth inhibition. The results suggest that metabolic products of ACOT11 and ACOT13 (14 to 18 carbon) are important regulators for lung adenocarcinoma. The findings are summarized in Fig. 6.

In conclusion, the results from the present study reported the role of ACOT11 and ACOT13 in lung adenocarcinoma. High ACOT11 and ACOT13 expression was associated with lung adenocarcinoma and poor overall survival rate. Knockdown of ACOT11 and ACOT13 significantly decreased cell proliferation, an effect that could be rescued by supplementing cells with free fatty acids. To the best of our knowledge, this is the first report regarding the potential oncogenic properties of ACOT11 and ACOT13 in lung adenocarcinoma. ACOT11 and ACOT13 may represent targets for novel treatments for patients with lung adenocarcinoma.

\section{Acknowledgments}

The present study was supported by grants from the Ministry of Science and Technology of the Republic of China (grant nos. MOST 103-2320-B-037-006-MY3 and MOST 104-2314-B-037-053-MY4), the KMU-KMUH Co-Project of Key Research (grant no. KMU-DK 105002 from Kaohsiung Medical University) and the Chi-Mei Medical Center and Kaohsiung Medical University Research Foundation (grant no. HSU 104CM-KMU-01).

\section{References}

1. Tennant DA, Durán RV and Gottlieb E: Targeting metabolic transformation for cancer therapy. Nat Rev Cancer 10: 267-277, 2010.

2. Faergeman NJ and Knudsen J: Role of long-chain fatty acyl-CoA esters in the regulation of metabolism and in cell signalling. Biochem J 323: 1-12, 1997.

3. Ellis JM, Bowman CE and Wolfgang MJ: Metabolic and tissue-specific regulation of acyl-CoA metabolism. PLoS One 10: e0116587, 2015.

4. Hunt MC, Tillander V and Alexson SE: Regulation of peroxisomal lipid metabolism: The role of acyl-CoA and coenzyme A metabolizing enzymes. Biochimie 98: 45-55, 2014.
5. Siegel RL, Miller KD and Jemal A: Cancer statistics, 2015. CA Cancer J Clin 65: 5-29, 2015.

6. World Health Organisation: World Cancer Report 2014. In: Chapter 5.1, 2014.

7. Wen T, Gao L, Wen Z, Wu C, Tan CS, Toh WZ and Ong CN: Exploratory investigation of plasma metabolomics in human lung adenocarcinoma. Mol Biosyst 9: 2370-2378, 2013.

8. Liu J, Mazzone PJ, Cata JP, Kurz A, Bauer M, Mascha EJ and Sessler DI: Serum free fatty acid biomarkers of lung cancer. Chest 146: 670-679, 2014.

9. Jung WY, Kim YH, Ryu YJ, Kim BH, Shin BK, Kim A and Kim HK: Acyl-CoA thioesterase 8 is a specific protein related to nodal metastasis and prognosis of lung adenocarcinoma. Pathol Res Pract 209: 276-283, 2013.

10. Barrett T, Wilhite SE, Ledoux P, Evangelista C, Kim IF, Tomashevsky M, Marshall KA, Phillippy KH, Sherman PM, Holko M, et al: NCBI GEO: Archive for functional genomics data sets-update. Nucleic Acids Res 41 (Database issue): D991-D995, 2013.

11. Stearman RS, Dwyer-Nield L, Zerbe L, Blaine SA, Chan Z, Bunn PA Jr, Johnson GL, Hirsch FR, Merrick DT, Franklin WA, et al: Analysis of orthologous gene expression between human pulmonary adenocarcinoma and a carcinogen-induced murine model. Am J Pathol 167: 1763-1775, 2005.

12. Su LJ, Chang CW, Wu YC, Chen KC, Lin CJ, Liang SC, Lin CH, Whang-Peng J, Hsu SL, Chen CH and Huang CY: Selection of DDX 5 as a novel internal control for Q-RT-PCR from microarray data using a block bootstrap re-sampling scheme. Bmc Genomics 8: 140, 2007.

13. Landi MT, Dracheva T, Rotunno M, Figueroa JD, Liu H, Dasgupta A, Mann FE, Fukuoka J, Hames M, Bergen AW, et al: Gene expression signature of cigarette smoking and its role in lung adenocarcinoma development and survival. PLoS One 3: e1651, 2008.

14. Okayama H, Kohno T, Ishii Y, Shimada Y, Shiraishi K, Iwakawa R, Furuta K, Tsuta K, Shibata T, Yamamoto S, et al: Identification of genes upregulated in ALK-positive and EGFR/KRAS/ALK-negative lung adenocarcinomas. Cancer Res 72: 100-111, 2012.

15. Selamat SA, Chung BS, Girard L, Zhang W, Zhang Y, Campan M, Siegmund KD, Koss MN, Hagen JA, Lam WL, et al: Genome-scale analysis of DNA methylation in lung adenocarcinoma and integration with mRNA expression. Genome Res 22: 1197-1211, 2012.

16. Kabbout M, Garcia MM, Fujimoto J, Liu DD, Woods D, Chow CW, Mendoza G, Momin AA, James BP, Solis L, et al: ETS2 mediated tumor suppressive function and MET oncogene inhibition in human non-small cell lung cancer. Clin Cancer Res 19: 3383-3395, 2013.

17. Győrffy B, Surowiak P, Budczies J and Lánczky A: Online Survival Analysis Software to Assess the Prognostic Value of Biomarkers Using Transcriptomic data in Non-Small-Cell Lung Cancer. PLoS One 8: e82241, 2013.

18. Kao YR, Shih JY, Wen WC, Ko YP, Chen BM, Chan YL, Chu YW, Yang PC, Wu CW and Roffler SR: Tumor-associated antigen L6 and the invasion of human lung cancer cells. Clin Cancer Res 9: 2807-2816, 2003.

19. Chu YW, Yang PC, Yang SC, Shyu YC, Hendrix MJ, Wu R and Wu CW: Selection of invasive and metastatic subpopulations from a human lung adenocarcinoma cell line. Am J Respir Cell Mol Biol 17: 353-360, 1997.

20. Livak KJ and Schmittgen TD: Analysis of relative gene expression data using real-time quantitative PCR and the 2(-Delta Delta C(T)) Method. Methods 25: 402-408, 2001.

21. Kirkby B, Roman N, Kobe B, Kellie S and Forwood JK: Functional and structural properties of mammalian acyl-coenzyme A thioesterases. Prog Lipid Res 49: 366-377, 2010.

22. Cohen DE: New players on the metabolic stage: How do you like Them Acots? Adipocyte 2: 3-6, 2013.

23. Hunt MC and Alexson SE: The role Acyl-CoA thioesterases play in mediating intracellular lipid metabolism. Prog Lipid Res 41: 99-130, 2002

24. Li Y, Song X, Zhao XJ, Zou LJ and Xu GW: Serum metabolic profiling study of lung cancer using ultra high performance liquid chromatography/quadrupole time-of-flight mass spectrometry. J Chromatogr B Analyt Technol Biomed Life Sci 966: 147-153, 2014.

25. Chang YS, Tsai CT, Huangfu CA, Huang WY, Lei HY, Lin CF, Su IJ, Chang WT, Wu PH, Chen YT, et al: ACSL3 and GSK-3 $\beta$ are essential for lipid upregulation induced by endoplasmic reticulum stress in liver cells. J Cell Biochem 112: 881-893, 2011. 
26. Hung YH, Chan YS, Chang YS, Lee KT, Hsu HP, Yen MC, Chen WC, Wang CY and Lai MD: Fatty acid metabolic enzyme acyl-CoA thioesterase 8 promotes the development of hepatocellular carcinoma. Oncol Rep 31: 2797-2803, 2014.

27. Pei Z, Fraisl P, Shi X, Gabrielson E, Forss-Petter S, Berger J and Watkins PA: Very Long-Chain Acyl-CoA Synthetase 3: Overexpression and Growth Dependence in Lung Cancer. PLoS One 8: e69392, 2013.

28. Adams SH, Chui C, Schilbach SL, Yu XX, Goddard AD, Grimaldi JC, Lee J, Dowd P, Colman S and Lewin DA: BFIT, a unique acyl-CoA thioesterase induced in thermogenic brown adipose tissue: Cloning, organization of the human gene and assessment of a potential link to obesity. Biochem J 360: 135-142, 2001.

29. Thorsell AG, Lee WH, Persson C, Siponen MI, Nilsson M, Busam RD, Kotenyova T, Schüler H and Lehtiö L: Comparative structural analysis of lipid binding START domains. PLoS One 6: e19521,2011.
30. Zhang Y, Li Y, Niepel MW, Kawano Y, Han S, Liu S, Marsili A, Larsen PR, Lee CH and Cohen DE: Targeted deletion of thioesterase superfamily member 1 promotes energy expenditure and protects against obesity and insulin resistance. Proc Natl Acad Sci USA 109: 5417-5422, 2012.

31. Kang HW, Niepel MW, Han S, Kawano Y and Cohen DE: Thioesterase superfamily member 2/acyl-CoA thioesterase 13 (Them2/Acot13) regulates hepatic lipid and glucose metabolism. FASEB J 26: 2209-2221, 2012.

32. Wei J, Kang HW and Cohen DE: Thioesterase superfamily member 2 (Them2)/acyl-CoA thioesterase 13 (Acot13): A homotetrameric hotdog fold thioesterase with selectivity for long-chain fatty acyl-CoAs. Biochem J 421: 311-322, 2009.

33. Kang HW, Kanno K, Scapa EF and Cohen DE: Regulatory role for phosphatidylcholine transfer protein/StarD2 in the metabolic response to peroxisome proliferator activated receptor alpha (PPARalpha). Biochim Biophys Acta 1801: 496-502, 2010 . 